\title{
Trans-splicing of pre-mRNA is predicted to occur in a wide range of organisms including vertebrates
}

\author{
Thomas Dandekar and Peter R.Sibbald \\ European Molecular Biology Laboratory, Postfach 1022 09, Meyerhofstrasse 1, D-6900 Heidelberg, \\ FRG
}

Received June 8, 1990; Accepted July 5, 1990

\begin{abstract}
Several known trans-splicing RNA structures were used to define a canonical trans-splicing structure which was then used to perform a computer search of the EMBL nucleotide database. In addition to most known transsplicing structures, many putative new trans-splicing sites were detected. These were found in a broad range of organisms including the vertebrates. Control experiments indicate that the search predicts known false positives at a rate of only $20 \%$. Trans-splicing may therefore be a very wide-spread phenomenon.
\end{abstract}

\section{INTRODUCTION}

When trans-splicing of pre-mRNA in trypanosomes was first reported [1] it was viewed as yet another peculiarity in an group of organisms already known to be atypical [2]. (For reviews of trans-splicing see [3-7].) The subsequent discovery of transsplicing in chloroplasts [8-14] and nematodes [7] indicated that the phenomenon was much more widespread. Naturally the question arises; how widespread is trans-splicing? This question is enticing not only for academic reasons. As Boothyroyd [15] has pointed out, many drugs that might control parasitic trypanosomes or nematodes also injure the host. If trans-splicing were to occur only in certain groups of organisms, (and particularly not in humans or cattle) then the trans-splicing reaction might provide an ideal target for novel drug therapies. The answer would of course also provide insight into the the more fundamental question of the evolution of trans and other forms of splicing [6].

While the $5^{\prime}$ mini-exon that is trans-spliced in kinetoplastids is sufficiently conserved [16] that it was possible to biochemically locate Crithidia fascuculata mini-exons using a $T$. Brucei probe [17], there is sufficient divergence between the mini-exon sequences of nematodes and trypanosomes [18] to prevent the location of nematode mini-exons using the same methodology. In general for phylogenetically distant species (which are the interesting ones) it will not be possible to use a mini-exon probe to biochemically screen novel species as a way of discovering if they also trans-splice. Since it is possible to identify cis-splicing sites using computer searches [19], it seemed that such an approach might also be used to locate trans-splicing sites. Studies on the mechanism of trans-splicing have identified many important features of trans-splicing RNA structures [6, 20]. By using such features to search DNA sequence data bases, we have been able to detect new putative trans-splicing sites and present evidence that trans-splicing occurs in organisms not previously known to exhibit trans-splicing.

\section{MATERIALS AND METHODS}

The EMBL nucleotide sequence database 22.0 [21] consisting of $38 \times 10^{6}$ base pairs and $32 \times 10^{3}$ sequences was used. Searches of both strands were performed on the EMBL VAX cluster using Pascal programs custom-written by one of us (TD) for the purpose.

The target for which to search was derived from the six transsplicing structures shown in Fig. 1. These structures were chosen because they are well documented [20] and relatively well understood. The canonical structure which was used as the target is shown schematically in Fig. 2. Features which were deemed obligatory were (1) the G-G doublet pairing with the Y-Y doublet; (2) a loop size of 3-10 nucleotides of which at least 3 must be U; (3) of the 4 positions following the G-G doublet, at least 3 of them must base pair with the opposite strand (here and elsewhere $\mathrm{G}: \mathrm{U}$ is considered a pair). If 1,2 and 3 pair then the stem is extended until only $50 \%$ of the bases (including positions 1,2 and 3) pair. If one of 1,2 or 3 do not pair then the stem is extended until a non-pair is encountered. (4) The distance ranges, $0-7$ bases are obligatory. (5) The Sm-site consists of a stretch of at least $3 \mathrm{U}$ interrupted by 0 or 1 other nucleotides, bracketed at both ends by the doublet R-R. The first nucleotide of the Smsite had to be within 60 nucleotides of the trans-splicing loop. (6) Stem loops I and II were identified with a simple energy scoring scheme. Each G:C pair scored 3, A:U scored 2, G:U scored 1 , and non-pairs scored -2 . A one nucleotide bulge was permitted and scored -2 . The resultant energy sum had to exceed both 6 and (the number of nucleotides in the stem plus loop divided by 2 , rounded down to the preceding integer).

Non-obligatory target features also contributed to the evaluation of putative hits (a hit is a positive located by a search). Each of the following seven possible features contributed one point; (1) the first residue after the G-G is a $\mathrm{U}$; (2) the second residue is an $\mathrm{A}$; (3) on the the other strand of the stem the $5^{\prime}$ most residue should be $\mathrm{G}$ or $\mathrm{U}$; (4) the $3^{\prime}$ adjacent residue is a $\mathrm{U}(5,6)$ the next two $3^{\prime}$ adjacent residues are $A$ or $U$; (7) there was no constraint on the next $3^{\prime}$ adjacent residue, but the one after should be a U. At least 6 of the 7 non-obligatory features were required. Note that the stem labelled as 'non-obligatory features' may 


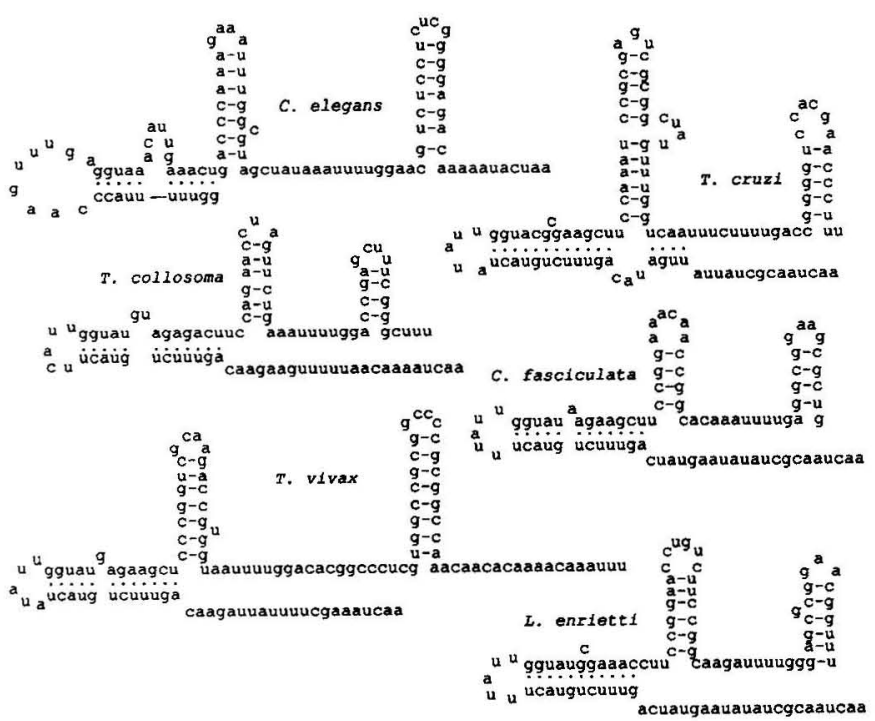

Figure 1. The 6 trans-splicing structures used to build the canonical structure, redrawn from [20]. These structures have been described in detail as follows, Caenorhabiditis elegans [18], Trypanosoma cruzi [16], Crithidia fasciculata [17], Leptomonas collosoma [1], Trypanosoma vivax [16], Leptomonas enrietti [29].
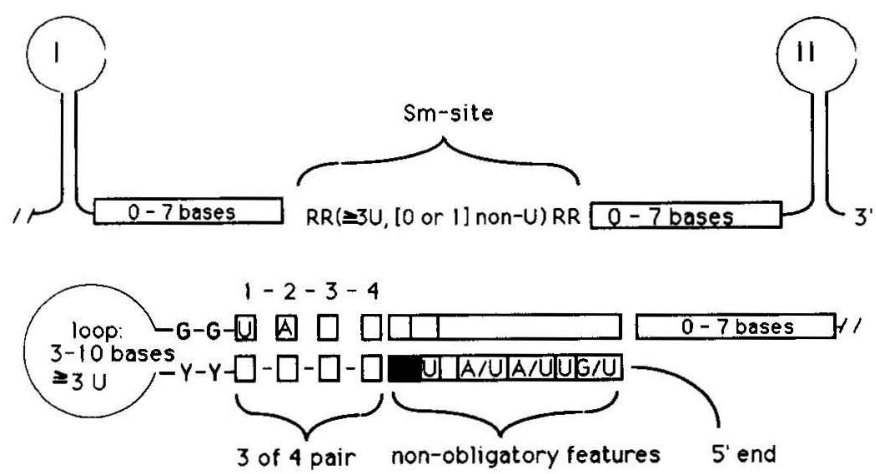

Figure 2. The canonical trans-splicing structure that was used as a target to perform searches. See Materials and Methods for details.

overlap with positions labelled 1 to 4 ( and its complementary strand) but the two have been drawn as non overlapping in the interests of clarity.

\section{RESULTS AND DISCUSSION}

There were 327 hits with 6 of the obligatory features and 37 hits with all 7. The distribution of the hits in various groups of organisms is summarized in Table 1. Detailed information about each individual putative trans-splicing site is presented in Table 2. Trans-splicing sites are predicted both in introns and exons with a trend to have more intron examples in higher organisms. Most sites are either known to be transcribed as RNA (EX, IN, LT in table $2 a$ and $2 b$; on (C) in Table $2 b$ ) or to exist as RNA as part of their life cycle (labeled as int in Table 2). It has been proposed that the DNA strand opposite a coding sequence also may often be transcribed [22] and many predicted trans-splicing sites are opposite CDS (Table 2).

\section{Controls}

Of the six sequences shown in Fig. 1 (those used to build the canonical structure) the five stored in the EMBL database were correctly identified and had all 7 non-obligatory structures $(L$. enrietti was not stored in the database as an unsplit motif.). In addition, several other known trans-splicing RNAs which were not part of the training set were correctly identified: LSMEDRNA (all idcodes refer to the EMBL database) and LSILINS1 from Leptomonas seymouri, TRSLRC and KTKPMC02 from T. cruzi; ALRLASL from Ascaris lumbricoides; CBRR5B, CBRR5A from Caenorhabditis briggsae; and CERR5 from Caenorhabditis elegans. In addition, numerous sites were identified in chloroplasts (Table 2) in which trans-splicing is known to occur [8-14]. All the known trans-splicing organisms including trypanosomes, Nematodes and Chloroplasts are correctly found by the search, usually with a score of 6 or 7 . However, some trans-splicing sites were not found and these are discussed next.

Trans-splicing RNAs from Trypanosoma brucei which have a diverged Sm-site, RRTCTRR [1] are not found (although KTKPCYB from $T$. brucei with a canonical Sm-site is found, Table 2). Use of the diverged T. brucei Sm-site makes the search quite non-specific (data not shown). Apart from this, trans-splice sites that are interrupted by introns, e.g. TCMXA or stored in the database as two or more parts such that the trans-splicing motif is split (e.g. L.enrietti, LESL1) are also not detected. Similarly, in some cases the GG of the trans-splicing structure is in the database but the up or down stream sequence does not appear in the sequence entry (e.g., CFMIEX, see also TCSLGB and TCSLGA which stop at the first G). As would be expected, such 'truncated' sequences are not identified by the search. Finally there are some known trans-splicing RNAs for which the exact site is not known experimentally and the search should allocate a splice site for these. Rps12 RNA in tobacco found by Koller et al. [12] to be trans-spliced, was missed. However a site was correctly predicted for the known trans-splicing of rps12 in liverwort; i.e., a site with 5 non-obligatory features is located 268 bases after rpL20 / rps 12 exon1 on the complementary strand at base 65539 in a long transcript [23]. In Chlamydomonas reinhardii psbB is known to trans-splice [13] but the search does not find a trans-splicing site. However the search does predict a trans-splicing site in psbC with 5 of the non-obligatory sites. Moreover, a trans-splicing site (with all 7 non-obligatory features) occurs in the Liverwort plastid at 42738, 14 base pairs upstream of $\mathrm{psbB}$ and another site occurs with 5 non-obligatory features in psbB (C) in tobacco at 39201. Four other sites in $C$. reinhardii have also 5 of the non-obligatory features and thus are excluded from Table 2 (only hits with 6 or 7 of the non-obligatory sites are presented). A complete list of the search results including hits with 5 of the non-obligatory features is available on request from TD. If the hits with a score of five are included, the search identifies all genera known to trans-splice which are stored as a complete motif in the data base. From chloroplasts it is known that both complete exons and small leader sequences are transspliced. Thus we did not restrict our search by demanding an additional homology to the small spliced leader as this hampers the identification of known chloroplast sites (data not shown).

Table 1 indicates considerable variation in the number of predicted sites per $10^{6}$ base pairs. From this it is tempting to conclude that trans-splicing is much more likely to occur in organelles, invertebrates and fungi than in the other groups. Caution is needed in making any such interpretation due to the presence of confounding factors. In particular, trypanosomes 
Table 1. The occurance of hits in various categories of DNA in EMBL 22.0. This database is divided, approximately along taxonomic lines, but primarily for convenience, into the categories: synthetic, viral, phage, organelles, prokaryotic, fungi, invertebrates, plants, vertebrates, mammals, rodents, primates, and unannotated. This Table shows the frequency of occurance of hits with 6 or 7 of the non-obligatory positions (strong positives) in each category. The total amount of DNA in base pairs and the number of sequences in each category are also shown. The rightmost column is the number of hits $(6+7)$ per 10 's'up $3(6)$ base pairs.

\begin{tabular}{lccccc}
\hline Category & sequences & base pairs & 6 point hits & 7 point hits & hits/106bp \\
synthetic & 755 & 274405 & 1 & 0 & 4 \\
viral & 2762 & 4502866 & 23 & 1 & 5 \\
phage & 512 & 613323 & 3 & 1 & 7 \\
organelle & 1550 & 2191964 & 52 & 16 & 6 \\
prokaryote & 3765 & 5301864 & 30 & 1 & 14 \\
fungi & 1455 & 2143702 & 31 & 0 & 15 \\
invertebrate & 2251 & 2714876 & 33 & 7 & 10 \\
plant & 1462 & 1988708 & 18 & 0 & 4 \\
vertebrate & 1563 & 1464464 & 14 & 0 & 6 \\
mammal & 1172 & 5763874 & 328 & 3 & 10 \\
rodent & 5591 & 6664208 & 57 & 6 & 10 \\
primate & 5466 & 2811783 & 27 & 37 & 10 \\
unannotated & 3195 & 38234565 & 327 & & \\
total & 31508 & & & 6 & \\
\hline
\end{tabular}

Table 2a. Putative splicing sites from the strand provided in EMBL 22.0. Each category of DNA is headed by the name of the category followed by the percentage of the database which the category constitutes, followed by the percentage of the total hits, followed by the ratio. This provides a course estimate of how frequent trans-splicing sites are relative to the amount of DNA being searched. After the heading the format is: EMBL idcode, position of GG in bases from the $5^{\prime}$ end of the sequence (counting only a,u,c,g,t), sequence title (which may have been abbreviated), and comments. Between the idcode and the position an asterisk ' $*$ ' indicates that all 7 of the nonobligatory features were found and that, as a consequence, this is a strong candidate for a transsplicing structure. The absence of an asterisk means that 6 of the nonobligatory features occurred. If the same sequence is cited in several data base entries, their idcodes are given directly after the sequence title. The following conventions were used for the comments: rep = repetitive DNA; GG: means the splice site is found exactly at the $5^{\prime}$ guanosine of the catalytical Guanosine doublette; test indicates that the trans-splice site is one of the ones used to build the canonical structure; ntst indicates a known trans-splicing RNA not from test set; knownO means that the organism is known to possess the trans-splicing reaction; knownS: known splice site; int: close to integration events; invA: before chloroplast inverted repeat $\mathrm{A}$; ptRNA: near tRNAs; cytB $=$ in cytochrome $B$; cox: in cytochrome oxidase subunit $1 ; \mathrm{PKC}=$ protein kinase $\mathrm{C}$; ori $=$ origine of replication; nuc = nucleoline; $\mathrm{km}=$ kanamycine resistance; $\mathrm{EX}(\mathrm{ORF}$ number $)$ : exon (open reading frame, number); IN (letter): intron(number); dn: after translation but before polyadenylation; pa: after polyadenylation site; ig: intergenic, no transcription unit. Fint,FinvA,FmRNA,F: five prime of int, invA, start of mRNA transcription; LT denotes a long RNA transcript occurring $3^{\prime}$ from the trans-splicing site. For references to individual sequences and annotation, refer to the EMBL database (Cameron 1988).

Synthetic

ECRGNABP $604 \quad$ E.coli rrn promotor/terminator fusion ig

fusion

Viruses

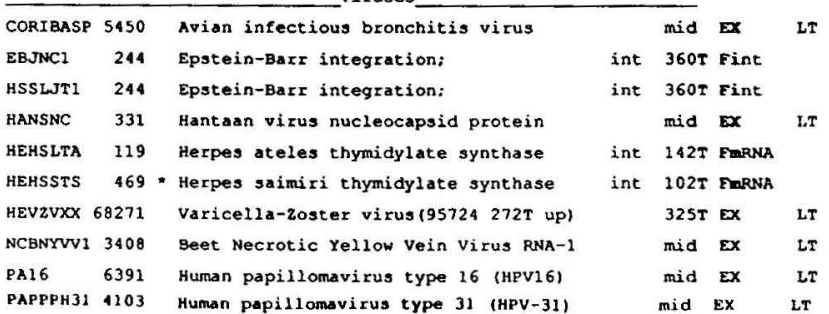

INPF3C01 1884 . Bacteriophage Pf3; (NewYork strain) BOF EX ORF 93
Organelles

ALCRDNA 3592 Astasia longa chloroplast ribos.DNA

CFRPME 416 * C.fasciculata mini-exon repeat

CHEGS16R 2277 Euglena grac. Chloroplast rRNA dupl.

CHHVPSBD 3221 Barley chloroplast psbB, H; petB, D

CHMPXX 69971 - Liverwort Marchantia chloroplast

CHMPXX 71381 (12 Trans5)

CHMPXX $109520 \quad$ (99837 ig)

CHNTXX 60343 Tobacco chloroplast; (5 Trans5)

CHNTXX 130104 (152057 PtRNA, ig)

CHOSXX 99488 Rice complete chloroplast genome

CHSARS16 166 mustard chloroplast rps16 gene

CHIATRN1 316 wheat chloroplast, URF 62, 5tRNA genes

CHZMRN4 28 Maize Arg- and Asn-trNA 3' region

KTKPCYB 508 T.brucei kplast apocyt.b

MITBO1 1100 "

KTKPMC02 609 T.cruzi kplast minicircle DNA PTC-21

LSMEDRNA 460 Leptom. seymouri mini-exon

LSIINS1; 782 "mini exon with insert element LINS1

MIBSRNAL 118 Boletus satanas mt large rRNA gene

MICAARS 1272 Cephalosporium acremonium mtDNA ARS

MIDMURFV 68 D.virilis mitochondriel DNA rRNA/URF

MTDYTRN 1173 D. Yakuba mitochondrial DNA

MILTRRNG 525 Leishmania tarentolae mtDNA(12S rRNA)

MINCND 260 Neurospora crassa me NADH dehydr.ase

MIPRGPL 3053 P. primaurelia me rDNA; 3053; 3053;

MISC13 7855 yeast cytochrome oxidase subunit1

MISC23: 522 as above

MISCCO1; 522 as above

MISPXX 5991 Sea urchin mt genome (Trans5 in NADH)

MITGTRN 196 Torulopsis glabrata tRe tenes

SCMTOR7A 51 reast (petite) mt replication origin

rCMIEx1 35 - Trypanosoma cruzi mini-exon repeat

TRSLRC 80 - T. Cruzi small spliced leader

TRSLRLC 77 . T, Leptom, coll. small splic. leader teat GG ig, not in rRNA

int $0 \mathrm{~T}$ int 2

cytB 20T up LT, 600

psbB S81T EX 1 knowno

CytB 43T up knowno

inva 140T Finva knowno

558F EX ORF 512 knowno

inve 100T FinvA knowno

100F dn ORF 23

290T up LT

PtRAI, EX URF62, LT, note1

ptRNA ig

EX, note2, knowno

EX, note2, knowno

ntst GG knowns

ntst $G G$ knowns

ntst GG knowns

ori ig

GG exactly at boundary

ori ig

90F EX

NADH $160 \mathrm{~T}$ up $2 T$, note?

ori

coxl mid IN aId text

$\operatorname{cox} 1210 \mathrm{EXX}$

ptRnA ig

ori ig

ceat $G$

ntat GG knowns

teat GG contro

\begin{tabular}{|c|c|c|c|c|c|c|}
\hline Ascrmo & 61 & $\begin{array}{l}\text { Prokaryotes } \\
\text { Acinetobact cycclohexanone monooxyg. }\end{array}$ & & $327 \mathrm{~T}$ & T FImRNA & \\
\hline ATACHS & 18611 & A.tumefaciens plasmid prils955 T-DNA & & S4T & EX ORF 23 & noted \\
\hline BSPRBHIK & $k 1510$ & Therm. bacillus plasmid proh1 (km) & $\mathrm{km}$ & $123 \mathrm{~F}$ & FX & $\mathbf{L T}$ \\
\hline CTORF & 2231 & C.trachomatis plasmid pCTT1: CTDNAB; & & mid & EX ORF2 & \\
\hline ECBIRA & 60 & E. coli biotin bira gene & & $238 \mathrm{~T}$ & T FmRNu & \\
\hline ECCPELC & 478 & Erwinia carotovora endo-pectate lyase & & $31 \mathrm{~T}$ & EMRNA & \\
\hline ECDMS & 6146 & E.coli anserobic dim. sulfoxide red. & & & ig & \\
\hline ECRRNBZ & 6674 & E. coli 165 rRNA, tRNA and two urfs & & & ig & \\
\hline HIOMPP 6 & 535 & H. Influenzae outer membrane protein P6 & & & ig & \\
\hline MVMCR1 & 2932 & Methanoc. voltae methyl-CoM reductase & & $228 \mathrm{~F}$ & F EX & \\
\hline PMMB 66EH & $=295$ & Plasmid pMAB66EH expression vector & & & ig & \\
\hline PSIRM & 498 & P.stuartil psti genes & & & ig & \\
\hline SAIIOKAR & 1510 & S.aureus plasmid pUB110dB (km) & $\mathrm{km}$ & $123 F$ & $\varepsilon \mathrm{Ex}$ & LT \\
\hline SAPS194 & 797 & S.aureus plasmid pS194 & & & ig & \\
\hline
\end{tabular}


SMTRNAI 389 Spiroplasma meliferum tRNAs; $389 ; \quad$ GG at 5'tRNA note3 vHCHIT 3179 V.harveyi N,N'-diacetylchitobiase; ig, 16 bp 3 , of 29 bp hairpin

\begin{tabular}{|c|c|c|c|c|}
\hline CAERGI 6 & 322 & Candid albicans cytochrome p-450 L1AI & $187 \varepsilon$ & Ex \\
\hline KLGAL & 3997 & Kluyveromyces lac. GALI, GAL7 and GALIO & $269 F$ & Ex Gal7 \\
\hline KLK1P & 3830 & Kluyv.lactis killer plasmid $\mathrm{kI}$ & 400F & EX ORFI \\
\hline KLK1P & 6365 & (killex toxin is ORF1): KLKILL05, KLKILL1L; & $150 \mathrm{~T}$ & EX ORFI \\
\hline SCDELI & 185 & Yeast delta and truncated delta element int & mid & int \\
\hline SCDP8 & 908 & Xeast delta-p8 gene 5 ' region & mid & $\mathbf{E x}$ \\
\hline SCHAP2 & 43 & Yeast transcriptional activator HAP2 & $97 \mathrm{~T}$ & up \\
\hline SCHOMMT & 121 & Yeast nuclear dna homologous to mt dna & & ig \\
\hline SCRPS31 & 935 & Yeast gene for ribosomal protein S31 & $120 \mathrm{~T}$ & note 10 \\
\hline SPMEI2 & 3328 & S.pombe mei2 gene & $604 \mathrm{~F}$ & pa \\
\hline SPTUBAI & 764 & S.pombe alpha-tubulin 1 & $383 E$ & $\mathbf{E x}$ \\
\hline & & Plants & & \\
\hline GMGY 3 & 2466 & Soybean glycinin subunit G3 gene & $140 \mathrm{E}$ & IN 3 \\
\hline GMTGMI & 2752 & Soybean lect in transposon $\mathrm{Tgml}$ & $800 \mathrm{~T}$ & int $P I$ \\
\hline LECHSOD & 134 & Tomato superoxide dismutase mRNA & $202 \mathrm{~F}$ & $\mathbf{E x}$ \\
\hline LHDEL & 6885 & Lilium del transposon (6531 EX) & $y$ at & the stop of \\
\hline МСРРCB & 1801 & M. crystallinum phosphoenolpyrcarb. & mid & IN 3 \\
\hline PSLECPGA & 225 & Pea PSL2 lectin pseudogene & mid & int \\
\hline STPATP1 & 1900 & Potato patatin pseudogene (SB6B) & mid & int \\
\hline STPATP2 & 5964 & Potato patat in pseudogene (SA1OC) & mid & int \\
\hline STWIN12G & 3431 & Poteto wound-induced genes WIN2 & $300 \mathrm{r}$ & mRNA \\
\hline 2MCPPS2G & 25 & Maize chloroplast psbG gene & $230 \mathrm{~T}$ & up \\
\hline 2MZEII9 & 3206 & Maize gene for Mr 29000 alpha zein & S4F & pa \\
\hline
\end{tabular}

Invertebrates

ALRGASL S44 - A.lumbricoides spliced leader

BMCH01 861 silkmoth chorion protein HC-B.13

BMCHRHCA 1745 silkmoth chorion protein HC-A.12/B.12

CBRR5B 336 * Caenorhabditis briggsae SS rRNA

CEACTL 352 - C.elegans actin spliced leader

DHMIF8A 3877 Drosophila hydei microcopia dhMif8

DHWHITE 13867 Drosophila white locus

NGRGE 799 N.gruberi 18 subunit rRNA gene

PCTHYSY 1267 P.carinii thymidylater synthase gene

PFANT2L 409 P.falciparum antigenic determinant

PFrSI 1361 P.falciparura repetitive DNA

pftrap 266 P.falciparum thrombospondin rel.prot.

ITCNJB 2114 Tetrahymena thermophila enjB gene
DMGPDHA 3902 Drosophila GP-dehydrogenase; DMGPDHG

TVMIEXI 35 * Trypanosome vivax mini-exon repeat

Primates (all hits are human)

HSB2M2 2113 Human beta-2-microglobulin gene 203T IN 3

HSC1A1 2428 Human alpha 1 collagen type I gene $56 \mathrm{~F}$ IN E

HSCRPG 1930 Human C-reactive protein; HSCRPGA; 500T dn

HSEB2CR2 3943 EBV receptor CX2 RNA; HSEBUR14; HSEBVR ; 100T dn

HSFIBEDA 2718 fibronectin gene ED-A region; $2719 \quad 278 \mathrm{~T}$ IN 2 note6

HSGA7331 1691 pancreas CA marker mRWA GA733-1;HSGA733A; $102 \mathrm{~T}$ dn

HSGASTB 479 Human gastrin gene, 3* region $400 \mathrm{~F}$ pa

HSHBEG 8493 Human LlHeg repetitive element int mid int

HSHBVINT 314 Human DNA / hepatitis B virus integr. int 600T Fint

HSHK2A 3726 - Human calcium-ATPase mRNA; HSCAATP4; $55 T$ dn LT

HSHMG14 650 non-histone protein HMG-14 mRNA 190F dn LT

HSHMGCOB 1140 * HMG COA reductase (EXI and promoter) mid IN 1

HSHSP90B 3862 * Human $90 \mathrm{kD}$ heat shock protein $23 \mathrm{~F}$ IN D

HSHTV2A 1028 Human tRNA-Val family

HSIFNB3 11512 Human interferon-beta-3 locus

HSIGVKA2 1910 Ig-kappa V(k)III pseudogene A22

PERNA $100 T$ FERNA

HSINSRC 5420 Human insulin receptor allele 1; 5420;

HSINSRD 5019 Human insulin receptor allele 2; 5019;

HSIRF2 1947 interferon regulatory factor-2 mRNA

HSMKDRW1 2246 MHC class II HLA-DRM53-bet.

HSPKCB1A 2152 protein kinsse $C$ beta I mRNA; HSPKCBI;

HSPROL1 478 Human prolactin gene 5' region

HSRBS 3976 Human retinoblastoma susceptib. mRNA

HSRSKPO8 311 Human kpni repeat mrna

HSTCGVAS 1044 T-cell receptor pseudogene; HSTCGVA;

HSUGRNA 152 Human gene for $U 6$ RNA

note1: Long mRNA, transcribed together with preceding psbc and psbD. Cleavage at the GG would yield a thylacoid membrane spansing peptide.

note2: Long mRNA; apocyt.b RNA is split and spliced in aspergillus and yeast; The URF s surzounding T.brucei apocye. b lack polyadenylation signals, but there is an $A-r i c h$ sequence at the 3 'end of a CDNA found by URF 2 probe.

note3: The 10 bp upstream of tRNA Pro together with the tRNA Pro form a TRANS6 which exact $1 y$ releases 5 'end tRNA Pro from the long five tRNA precursor.

note 4: The TRANS starts at bp 18616 and ends exactly at the end of ORF (bp18687).

note5: The two alleles have slightly different TRANS, but at the same position.

note6: EX2 before IN2 is untranslated in liver and alternatively opliced mRNAs with and without Ex2 occur in difforent ratios in different tissues.

note7: Longer RNA species of $5,5.6$ and $7 \mathrm{~kb}$ are only lit by probes 5 ' to the nd4I gene or exon probes but not by intron or downstream probes.

note8: A trans-splicing event would load to the shortened $4 \mathrm{~kb}$ mRNA observed in retinoblastomes.

note9: Identical positions. Parasite under genetic pressure 1ike Trypanosomos!

note10:Entire 3 'end $(128$ bp) of protein $\$ 31$ mRNA forms a trang-splicing RNA.

Vertebrates

CIACTB 3965 Grass carp beta-actin gene

GGEF9E3 898 Chicken embryo fibroblast protein mRNA

$6435 d \Omega$

$284 \mathrm{~T}$ dn $\mathrm{LT}$

mid IN 2

$\begin{array}{lll}\text { GGMYC } 3384 & \text { Chicken cellular myc onc.gene; GGCMYCA } \\ \text { GGMYHE } 8908 \text { Chicken embryonic myosin heavy chain }\end{array}$

GGPGR 4270 Chicken progesterone receptor mFNA

GGRSVIND 878 Chicken RSV-transformed mRNA

GG exactly at IN13/EX14

$30 \mathrm{~T} \mathrm{dn}$

$400 \mathrm{~T}$ dn

$56 \mathrm{~T}$ IN 1

XLENK02 40 Xenopus laevis proenkephalin gene A2

$100 \mathrm{~T} \mathrm{dn}$

Marma Is

CHEBGLII 1019 Goat apsilon II beta-globin gene $\quad 90 F$ IN 2

OCPKCer 2237 Mabbit protein kinase $C$ beta mRNA PKC $7 F$ dn

\begin{tabular}{|c|c|c|c|c|c|c|}
\hline DOREPI & 2778 & Kangaroo rat repetitive DNA: 2779; & int & $516 \mathrm{~T}$ & int & $\mathbf{L T}$ \\
\hline MAHPRT & 1083 & Chinese hamater hprt mus & & $60 \mathrm{~T}$ & dn & \\
\hline MMBGLOFG & 45 & Mouse downst ream of beta-globin gene & & $600 \mathrm{E}$ & pa & LT \\
\hline MMDHF 4 & 781 & Mouse mutant tetrahydrofol. -DH mRNA & & $150 \mathrm{~T}$ & an & \\
\hline MMDHF7 & 325 & Mouse dihydrofolate reductase exon 3 & & $10 \mathrm{~T}$ & IN 2 & 2 , near \\
\hline MMETNB & 2272 & Mouse early transposon (ETn) & int & $30 \mathrm{~T}$ & Fint & \\
\hline MMIRF12 & 2192 & Mouse interferon reg. factor -2 mRNA & & $243 \mathrm{~T}$ & $d n$ & LT \\
\hline MMNUCLO1 & 1906 & Mouse nucleolin gene: & nuc & $=290 \mathrm{~T}$ & IN 1 & \\
\hline MMPP10SR & 3880 & Mouse retinoblastora susceptib.; 3885 ; & & $1020 \mathrm{~F}$ & dn & \\
\hline MMTICPS & $2003 *$ & Mouse Ilc pseudogene for MHCI antigen & & 2395 & IN 3 & \\
\hline RNCAMI 3 & 1915 & Rat CaMI gene for calmodul in & & mid & IN 3 & \\
\hline RNFBAG & 3420 & Rat gene for alpha-fibrinogen & & $573 \mathrm{~T}$ & IN 1 & \\
\hline RNNUCLEO & 2064 & Rat nucleolin gene, exons 1 and 2 & nuc & $=290 \mathrm{~T}$ & IN 1 & \\
\hline RNPECOA & 1509 & Rat peroxisomal enOy1-COA mRNA & & $578 \mathrm{~T}$ & EX & \\
\hline RSLIN4A & 3033 & Rat long interspersed repet. DNA & int & mid & int & \\
\hline
\end{tabular}

Table b. Trans-splicing sites from the strand complementary to that given in EMBL 22.0. The position is where the GG occurs in terms of the strand given in EMBL 22.0; it is not the distance from the 5 ' end of the complementary strand. Legend, as in Table $2 \mathrm{a}$ with the addition that CDS is coding sequence and opp means opposite from the strand searched. On (C) means that the transcription unit is here, on the complementary strand.

\begin{tabular}{|c|c|c|c|c|c|}
\hline & & $\begin{array}{l}\text { Synt het ic (no hita) } \\
\text { viruses }\end{array}$ & & & \\
\hline ADLE2B & 1944 & Adenovirus type 12 E2b region DNA & pol mid & Ex & on $\langle c\rangle$ \\
\hline IRIEPEH & 3190 & Insect iridescent virus type 6 & rep & & \\
\hline PXVACLEM & 805 & Vaccinie virus transposition mutant & int & & \\
\hline REMULVT 3 & 147 & Murine leuknemia virus (MuLV) 3'LTR & ret roviru & & \\
\hline REMULVTS & 432 & Murine Laukemia virus (MuLV) S'LTR & ret roviru & & \\
\hline RESPUENV & 377 & Human spums ret rovirus & ret roviru & & \\
\hline COTGEV3 & 2941 & Enteric coronavirus & opp non-s & structr. & protein \\
\hline CORTGEVM & 1020 & Enteric coronavirus & opp nucle & ocapsid & protein \\
\hline HRVVP2 & 864 & Muman rotavirus & opp up2 & & \\
\hline PARPVCO1 & 3471 & Canine parvovirus & opp vp2 & & \\
\hline PARPVCCP & 419 & Canine parvovirus & opp CDS & & \\
\hline PARPVCVP & 1799 & Canine parvovirus & & & \\
\hline MCACGDH & 64 & Cauliflower mosaic virus & & & \\
\hline VSVNJMPA & 195 & $\begin{array}{l}\text { Vesicular stomatitis virus N protein } \\
\text { Phages }\end{array}$ & opp CDS & & \\
\hline YOVP1 & 542 & Bacteriophage P1 IS2 insertion hot spot & int $21 F$ & ORF1 & on $(\mathrm{C})$ \\
\hline STSPOZ & 486 & Bacteriophage SP01 with cerminal repeat & GG start & EX $9 p^{28}$ & on $(C)$ \\
\hline
\end{tabular}


Organelles

CHCERR23 759 Chlorella ellipsoidea plastid

CHMPTRN 2960 * Liverwort Marchantia plastid; knowno

CHMPXX 46949 Liverwort Marchantia plastid; knowno

CHMPXX 30617

CHMPXX 12730 - very good candidate!! knowno

CHOSXX 115630 Rice complete chloroplast genome betweer

CHOSXX 103186 Rice complete chloroplast genome

CHOSXX 49441 Rice complete chloroplast genome

CHZMNDH 263 Maize chloroplast ndhD, ndhe and psaC

MIDMM2 124 - D.melanogaster mt large rrna gene;

MIDHTRN 7367 - Drosophila mt DNA;

MIDYRRN 15258 Drosophila yakuba me DNA

MIMM01 1475 Mouse mtDNa

M1NCND2D 801 Maurospore crassa mt DNA duplications

MIPAIVS2 961 Podospore enserina class II intron

MIRC12S 1869 Rana cateoboiana mtDMA

MIRNRN 280 Rat mtDNh; MIRNXX

MISC13 2283 Youst cytochrome axidase subunit 1

MIscco12 2656 Yeast cytochrome oxil gene and flanks

MISCORIK 281 Yaast mitochondrial ori2-ori7 region

MI TBCOX 1903 - Irypanosoma brucei mt cyt $c$ oxidase

MITOMM 1351 Mouse mitochondrial genome

MITOMM 10410 Mouse mitochondrion

MIXLG 3343 Xenopus laovis mt genome : XIMTDTG

MIXLORI $866 \quad$ X.laevis mt ori

CHNTXX 90472 Tobacco plastid

MIBTXX 4916 Bovine mitochondrion

TBGP01 571 I.brucei surface protein

Prokaryotes

BAMPR 322 * B. amyloliquifaciens alk. protease

BSPRBHIK 198 Thermophile kanamycin plasmid

between reps

BSREPB 198 Bacillus plasmid

BSRODC 3662 Bacillus subtills rodC operon

ECCE12 75 Exwinia ehrysantheni endoglucanase

opp cos

MMCR 1360 Methanococcus van.

MVRPOP 6666 Methanococcus van.

NGTIA 72 N.gonorrhoese eransformation inhibitory DNA

SALS4BOP 220 S.aureus phage L54 attL site

SAPUBI10 2843 S.aureus plasmid

SMPAC 4797 Strepto.matans

SMRPLKA 214 Serratin marcesc.ribos protein L11, L1 opp cDS opp rpL15

in neo( $x$ ) CDS

Fungi

DDAAC11 975 Dictyostelium discoiedeum AAC-rich mRNA

DDACTA32 731 Dictyostelium discoiedeum actin

SCADE3 4176 Saccharomyces cerevisiae C-1-tetrahydrofolate synthase: opp CDS

SCBAF1 1426 Saccharomyces cerevisiae transcription factor Baf1; Opp CDS

SCCPA1 1535 Saccharomyces cerevisiae carbamoyl-phosphate synthetase; opp CDS

SCGCDI 1438 Saccharonyces cerevisiae GCDI gene;

SCMAL28C 1961 SAccharomyces cerevisiae mutant, mal2-8cp gene

opp cDs

SCMLL6R 1429 Saccharomyces cerevisiae MAL6R gene;

SCMAT4 294 Saccharonyces cerevisise mating type;

SCMYO1 592 Saccharomyces cerevisise myosin-like cdc protein; opp cDS

SCPDC1 1401 Saccharomyces cerevisiae pyruvate decarboxylase: opp CDS

SCRADSO 3032 Saccharomyces cerevisiae RADSO gene; in heptad repeat region

SCRAR1 1869 Saccharomyces cereviside RARI gene;

SCSERS 1432 Saccharomyces cerevisiae sery1-tRNA synthetase;

opp gene

SCSILA 244 Saccharonyces cerevisiae silencer DNA;

SCSIR2 255 Saccharomyces cerevisiae mating type control):

ASPHT 3 A 231 Avena sativa phytochrome

GMLEA 843 SOybean lectin

HVLEU 911 Barley thiol protease

IBGSPOAL 433 Sweet potato sporamin $A$

PSELIP 1702 Pea plastid early-light-induced protein chloroplast nuclear

encoded

SCNACT 2112 S.cerevisiae N-acetyltransferase

opp CDS

vFVICG 2885 . vicia faba vicilin gene

Invertebrates

CBRRSA 586 * Caenorhabditis briggsae 59 rRNA (1kb) knowno in spliced leader:

CERR5 210 . Caenorhabditis elegans DNA for SS IRNA knowno in spliced leader!

CETUBUB 1631 Caenorhabditis olegans beta-tubulin knowno

DMANTPEB 497 D.melanogaster antennapedia; DMANTPRA

DMIS176 6632 D.melanogaster copia-1ike element 17.6 int

DMIS297 6286 D.melanogaster transposable element 297 int
OMLGL2 5099 D.melanogaster giant larvae; int these two sites

DMLGL2 2293 D.melanogaster giant larvae; int are identical

DMRT412G 2740 Drosophila retrotransposon 412; $\mathrm{gg}$ at 2754 is also a site

DNTN10P 2665 D.nebulosa transposon N10 int

PFIRAA 229 P.falciparum interspersed repeat antigen int

DML-2AMD 943 Drosophila $\alpha$-methyldopa hypersensitivity

DMESPLM7 217 * enhancer of split

DMUBXGS 2679 ultrabithorax promoter

PCMSA 755 P.chabaudi merozoite antigen opp surface antigen CDS

PFSA27 2614 Plasmodium falciparum S-antigen upstream of poly rep region

PYCSP 281 P.yoelii eireum-sporozoite opp CDS

PYCSP1 $281 \quad$ P.yoelii circum-sporozoite opp CDS

SPCAX 031 Strongy locentrotus, purpuratus collagen IV opp intr

тTH101 1970 Tecrahymena HA-I gene and flanks

GGERBaF 2299 Chicken C-Erb oncogenic ALV insertion; int

GGC1k225 525 Chicken alpha-2 collagen 1

GGOVAL 6224 Chicken ovalbumin gene:

GGPEC 310 Chicken ppanolpyr, carboxykinase

opp intron G

Mammals

BTNABGSA 1307 Bovine galactogyltransf.

upstream of coding region

OCIL1R 1958 Rabbit interleukin I pre-cursor

OCPRGS 734 Rabbit progesterone receptor

SSAPOB2 3621 Pig apolipoprotein B opp exon

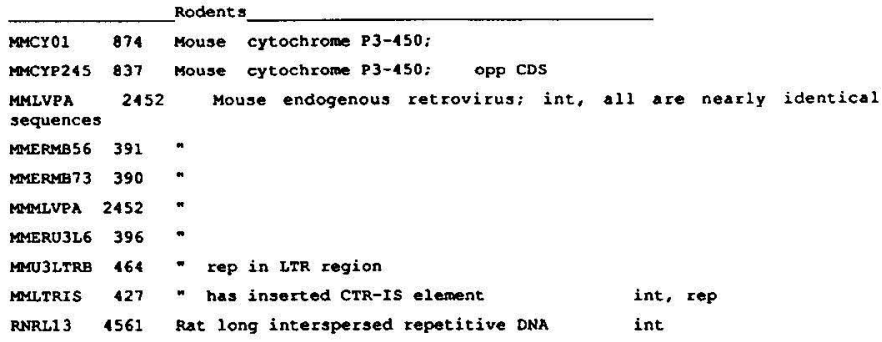

Mirfi2 353 Mouse interferon regulatory factor-2 opp $\cos$

MDM1A 1248 * Mouse mdm-1 gene

MARPL3A 157 Mouse ribosomal protein 132

MMTPMYOB 743 Mouse beta-tropomyosin

RNCYP45I 6878 Rat cytochrome P450IIE1;

RNLCAGI 9687 Rat leukocyte common antigen;

RNLCAR 483 Rat leukocyte common antigen;

int near processed gene opp cDS

RNURIM 1292 Rat uricase

opp CDS

opp pot. glycosylation site

Primates

GCGAL32 153 G_crassicaudatus short repeated DNA int

HTLV1RES 589 Human HTLV-I related retroviral sequence: int

HSARGi 162 Human arginase

HSCALL01 5467 Human lymphoblastic leukemia antigen:

HSCN2 100 Human skin collaganase:

HSCN25 166 Human synovial collagenase

HSCOLLA 178 Human collagenase

HSCYPJ 1613 Human eytochrome P-4SOj:

HSENKPH2 140 Muman enkephalin gene:

HSFBRA 695 Human fibrinogen a-alpha-chain;HSFBRMA opp CDS

HSFBRGG 8575 Hurian fibrinogen game chaini HSFBRGAB

HSFIB1 1894 * Human fibronectin;

HSGASTA 4231 Human gastrin gene;

HSGCRER 2823 Huan beta-glucocorticoid receptor;

HSHLASBA 13320 Human HLA-SB (DP) alpha gene

HSHLASBA 12953 Human HLA-SB(DP) alpha gene

HSMLCAB 900 Human alk. myosin light chain 1:

HSMLClF 900 Huran alk. myosin light chain 1

HSMLCAC 773 Human alkali myosin light chain 3:

HSNMYC 6702 Human n-myc gene;

HSNMYCO 1949 Human n-myc gene:

HSNAYC 350 Human n-myc gene;

HSOTC 282 ornithine transcarbamylase;

HSUG4PA 497 * U4 small nuclear RNA pseudogene opp non-translated mR.. opp CDS opp cDS

opp non-translated mRWA

int, Alternative splicing! int (near Alu)

int

Opp non-coding mRNA

opp CDS

opp intron

opp CDS 


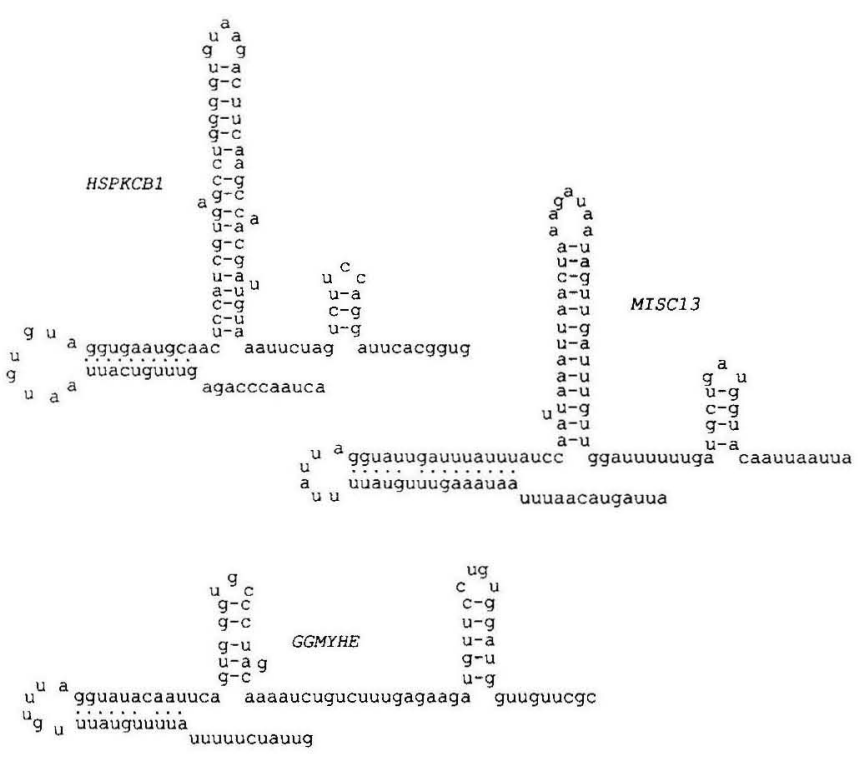

Figure 3. Three strong candidates for newly identified trans-splicing sites. The secondary structures of these predicted sites are very similar the known sites shown in Fig. 1 HSPKCB1 is human protein kinase C, MISC13 is yeast mitochondrial, and GGMYHE is chicken embryonic myosin heavy chain. None of these organisms are known yet to trans-splice in vivo.

appear to trans-splice every pre-mRNA $[3,6,16,24]$ and so the observation that there are many more splice sites per unit sequence in trypanosomes may be simply because they splice a higher fraction of their pre-mRNA than do other organisms. Secondly, there is a tendency in molecular biology to sequence DNA related to previously sequenced DNA and whether this is due to the availability of particular probes or common interest in certain sequences, the result is that the database does not consist of 'independent events'.

The search was able to distinguish between trans-splicing sites and other types of RNA (Table 2). In no cases did the search mis-identify tRNA as a trans-splicing site (there is however a plausible site which would release tRNA (proline) from a long precursor in Spiroplasma, SMTRNA1). Only in two cases ( $N$. gruberi $18 \mathrm{~S}$ rRNA, and L. taraentolae mitochondrial 12S rRNA) were rRNA genes apparently mistaken for trans-splicing sites and no small nuclear RNA known to participate in cis-splicing was confused with a trans-splicing site. Both cis- and transsplicing RNAs have several similar features, but perform different biological functions and the search discriminates between them. These three negative controls for RNA structures which appear similar but which are functionally distinct underline the ability of the search to pick good candidate sites for trans-splicing.

In an attempt to estimate the background error rate of the search, the search was repeated on the strand in the EMBL database with the sequence $\mathrm{YY}(\geq 3 \mathrm{C}, \pm 1$ non $\mathrm{C}) \mathrm{YY}$ substituted for the Sm-site. This motif should be biological nonfunctional as its $\mathrm{Sm}$-site is destroyed [20]. This search found $12 \%$ as many 'hits' with 6 or 7 of the nonobligatory features as did the search using the correct Sm-site. We estimate therefore that as many as $80 \%$ of the putative hits in Table 2 may be real. Similarly, since it is known from $T$. brucei that other transsplicing sites can exist with diverged Sm-sites, and possibly with other variations of which we are as yet unaware, the search is almost certainly incomplete.
Phylogenetic distribution of predicted trans-splicing structures

Even taking into account that $20 \%$ of the putative hits in Table 2 might be false positives, there is still considerable evidence that trans-splicing occurs in several groups of organisms in which this mechanism has not been previously identified. There are groups in which it is not predicted such as the mycoplasmas but this may well be due to the small amount of mycoplasma DNA in the database. Despite the availability of $38 \times 10^{6}$ base pairs, this is actually a very small sample for this type of study. However, in general, the phylogenetic distribution of predicted trans-splicing structures is sufficiently broad to suggest that transsplicing is quite primitive. The question has been raised as to whether trans-splicing is an unusual type of splicing that evolved in trypanosomes and a few other organism as an adaptive feature [6]. We would argue that trypanosomes did not develop transsplicing as an adaptation but that they have retained it .

We observe that in a high number of cases (40), the predicted trans-splicing site is proximal to an integration site and that in an additional 12 cases, the predicted trans-splicing site is in or proximal to repetitive elements or transposons. Other workers $[25,26]$ have observed an association between retroposons and mini-exons. This association of trans-splicing structures and integrating DNA may synergistically accelerate the spread of both but perhaps also contributes to the recombination of protein coding regions originally carried by the RNA having the respective trans-splicing site.

\section{Strong candidate sites}

It seems appropriate to identify some particularly strong candidates for experimental testing. In particular, MIDMURFV, SMTRNA1, LHDEL, PCTHYSY, GGMYHE, HSPKCB1A, and HSTCVA5 contain the catalytic double guanosine [20] exactly at an exon boundary. The oxi3 locus in yeast (MISC13) has a well formed predicted trans-splicing site in the intron aI4. The intron is already known to be important for splicing [27] and the following self-splicing group II intron aI5g could be divided in vitro to yield two RNAs that trans-spliced in vitro with associated trans-branching of excised intron fragments [28]. Refer to Fig. 3 for secondary structure diagrams of three of these strong candidate sites. Comparison of these with the structures in those in Fig. 1 shows how highly similar in structure they are to known sites. More examples are given in Table 2; particularly striking are cases in which a trans-splicing site is found in similar positions in the same gene from different organisms (class in Table 2) or additional evidence is available (Table 2 and notes to Table 2).

\section{CONCLUSION}

The search identified the five trans-splicing structures from the test-set which are undisrupted in the EMBL database and detected (with correctly predicted trans-splicing sites) all groups known to trans-splice, including Trypanosomes, Nematodes and Chloroplasts. Neither rRNAs (two exceptions), tRNAs nor small nuclear RNAs involved in cis-splicing were mistakenly identified as trans-splicing sites. The search could not identify every known trans-splicing site from every species (diverged T.brucei sites, two known Chloroplast trans-splicing RNAs and truncated transsplicing sites in data base entries were missed). Other RNA structures also could promote trans-splicing and might not have been been detected. It is also possible that some putative sites are in fact pseudogenetic in nature. However, enough new 
candidate trans-splicing sites (even taking into account a background estimate of $20 \%$ false positives) have been detected to suggest that trans-splicing may be much more wide spread then previously thought. There are several good candidate structures identified in species not yet known to possess transsplicing available for experimental testing (Table 2), including sites from vertebrates.

\section{ACKNOWLEDGMENTS}

We thank Angus Lamond, David Tollervey and Benjamin Blencowe for reading the manuscript and making suggestions. PRS is grateful to the National Sciences Engineering and Research Council of Canada and the Alexander von Humboldt-Stiftung for financial support. TD wishes to thank Boehringer Ingelheim Funds for Basic Medical Research for support.

\section{REFERENCES}

1. Milhausen, M., Nelson, R.G., Sather, S., Selkirk, M. and Agabian, N. (1984) Cell 38, 721-729.

2. Sharp, P.A. (1987) Cell 50, 147-148.

3. Borst, P. (1986) Ann. Rev. Biochem. 55, 701-732.

4. Van der Ploeg, L.H.T. (1986) Cell 47, 479-480.

5. Braun, R. (1986) Bioessays 5, $223-227$.

6. Laird, P.W. (1989) Trends. Genet. 5, 204-208.

7. Nilsen, T.W. (1989) Exp. Parasitol. 69, 413-416.

8. Ohyama, K., Fukazawa, H., Kohchi, T., Shirai, H., Sano, T., Sano, S., Umesono, K., Shiki, Y., Takeuchi, M., Chang, Z., Aota, S., Inokuchi, H. and Ozeki, H. (1986) Nature 327, 572-574.

9. Shinozaki, K., Ohme, M., Tanaka, M., Wakasuigi, T., Hayashida, N., Matsubayashi, T, Zaita, N., Chungwongse, J., Obakata, J., YamaguchiShinozaki, K., Ohto, C., Torazawa, K., Meng, B.Y., Sugita, M., Deno, H., Kamogashira, T., Yamada, K., Kusada, J., Takaiwa, F., Kato, A., Tohdoh, N., Shimada, H. and Sugiura, M. (1986) EMBO J. 5, 2043-2049.

10. Umesono, K., Ozeki, H. (1987) TIG 3, 281-287.

11. Zaita, N., Torazawa, K., Shinozaki, K. and Suguira, M. (1987) FEBS Lett. 210, $153-156$.

12. Koller, B., Fromm, H., Galun, E. and Edelman, M. (1987) Cell 48,111-119.

13. Kuck, U., Choquet, Y., Schneider, M., Dron, M. and Bennoun, P. (1987) EMBO J 6, 2185-2195.

14. Hiratsuka, J., Shimada, H., Whittier, R., Ishibashi, T., Sakamoto, M., Mori, M., Kondo, C., Honji, Y., Sun, C.R., Meng, B.Y., Li, Y.Q., Kanno, A., Nishizawa, Y., Hirai, A., Shinozaki, K. and Sugiura, M. (1989) Mol. Gen. Genet. 217, $185-194$.

15. Boothroyd, J.C. (1985) Ann. Rev. Microbiol. 39, 475-502.

16. De Lange, T., Berkvens, T.M., Veerman, H.J.G., Carlos, A., Frasch, C., Barry, J.D. and Borst, P. (1984a) Nucl. Acids Res. 12, 4431-4443.

17. Muhich, M.L., Hughes, D.E., Simpson, A.M. and Simpson, L. (1987) Nucl. Acids Res. 15, $3141-3153$.

18. Krause, M. and Hirsh, D. (1987) Cell 49, 753-761.

19. Senpathy, P., Shapiro, M.B. and Harris, N.L. (1990) Meth. Enzymol. 183, $252-278$.

20. Bruzik, J.P., Van Doren, K., Hirsh, D. and Steitz, J.A. (1988) Nature 335, $559-562$.

21. Cameron, G.N. (1988) Nucl. Acids Res. 16, 1865-1867.

22. Tramontano, A., Scarlato, V., Barni, N., Cipollaro, M., Franze, A., Macchiato, M.F. and Cascino, A. (1984) Nucl. Acids Res. 12, 5049-5059.

23. Kohchi, T.,Ogura, Y., Umesono, K., Yamada, Y., Komano, T., Ozeki, H. and Ohyama, K. (1988) Curr. Genet. 14, 147-154.

24. De Lange, T., Michels, P.A.M., Veerman, H.J.G., Cornelissen, A.W.C.A and Borst, P. (1984b) Nucl. Acids Res. 12, 3777-3789.

25. Affoter, M., Rindisbacher, L. and Braun, R. (1989) Gene 80, 177-183.

26. Aksoy, S., Lalor, T.M., Martin, J., Van der Ploeg, L.H.T. and Richards, F.F. (1987) EMBO J. 6, 3819-3826.

27. Dujardin, G., Jacq, C. and Slonoimski, P.P. (1982) Nature 298, 628-632.

28. Jarrell, K.A., Dietrich, R.C. and Perlman, P.S. (1988) Mol. Cell Biol. 8, 2361-2366.

29. Miller, S.I., Landfear, S.M. and Wirth, D.F. (1986) Nucl. Acids Res. 14, $7341-7360$. 